\title{
INTRODUCTION Applications of logical approaches to argumentation
}

\author{
João Leite ${ }^{\mathrm{a}}$, Tran Cao Son ${ }^{\mathrm{b}}$, Paolo Torronic* (D) and Stefan Woltran ${ }^{\mathrm{d}}$ \\ ${ }^{a}$ NOVA-LINCS, Universidade Nova de Lisboa, Campus de Caparica, 2829-516 Caparica, Portugal; \\ ${ }^{b}$ Computer Science Department, New Mexico State University, Las Cruces, NM 88003, USA; ${ }^{c}$ DISI, \\ Università di Bologna, Viale del Risorgimento 2, 40136 Bologna, Italy; ${ }^{d}$ Institute of Information Systems, \\ Vienna University of Technology, Favoritenstraße 9-11, Vienna, Austria
}

\section{Preface}

The fourteenth International Workshop on Computational Logic in Multi-Agent Systems (CLIMA XIV) was held from 15-18 September 2013 in Corunna, Spain, and was co-located with the 22nd International Conference Logic Programming and Non-Monotonic Reasoning (LPNMR) and the 7th International Workshop on Modular Ontologies (WoMO'13). The purpose of the CLIMA workshops is to provide a forum for discussing techniques, based on computational logic, for representing, programming and reasoning about agents and multi-agent systems in a formal way.

Other CLIMA editions have mostly been conducted in conjunction with major computational logic and artificial intelligence events such as CL in 2000, ICLP in 2001 and 2007, FLoC in 2002, LPNMR and AI-Math in 2004, JELIA in 2004 and 2008, AAMAS in 2006, MATES in 2009, ECAI in 2010, 2012, and 2014, and IJCAI in 2011. In 2005, CLIMA VI was organised as a stand-alone event. General information about the workshop series, with links to past and future events, can be found on the CLIMA workshop series home page. ${ }^{1}$

Driven by the fact that argumentation has become an important and exciting topic in artificial intelligence, intimately related to both the areas of computational logic and of multi-agent systems, CLIMA XIV featured a Special Session on Argumentation Technologies organised by Paolo Torroni and Stefan Woltran. Recent years have witnessed strong research activities ranging from theory to applications and increased uses of argumentation throughout a variety of subdisciplines. The Special Session on Argumentation Technologies was intended to bring researchers from these communities together, providing a forum to discuss concepts, theories, methodologies, and applications of computational models of argumentation.

CLIMA XIV received the exceptionally high number of 44 submissions, of which 23 were accepted for publication in the Springer Proceedings (LNCS 8143). In particular, 21 of these submissions were attracted by the Special Session on Argumentation Technologies.

The number and overall high quality of the papers on argumentation technologies led to the decision to edit a volume dedicated to this topic, resulting in this special issue of Argument \& Computation on applications of logical approaches to argumentation. Another special issue on selected papers covering all areas of CLIMA is to be published in the Journal of Logic and Computation.

*Corresponding author. Email: paolo.torroni@unibo.it 


\section{Contents}

This special issue contains the following extended and carefully reviewed versions of select contributions to CLIMA XIV.

In 'Automatic evaluation of design alternatives with quantitative argumentation', Pietro Baroni, Marco Romano, Francesca Toni, Marco Aurisicchio, and Giorgio Bertanza present a novel approach for argumentation in engineering design. To this end, they propose to extend the well-known issue-based information system method by numerical information in order to increase the quality in the decision-making process. The resulting formalism is called Quantitative Argumentation Debate frameworks and an implementation thereof is evaluated in the context of three different case studies.

'Context-aware reconfiguration of large-scale surveillance systems: argumentative approach' is also an excellent example for the successful application of argumentation in practical domains. In fact, Peter Novák and Cees Witteveen introduce here the METIS system prototype developed for supporting maritime safety and security by facilitating continuous monitoring of vessels in national coastal waters. In the paper, they propose a continuous reconfiguration algorithm for their system that relies on standard results from abstract argumentation and corresponds to the computation of a grounded extension.

The contribution by Ofer Arieli and Christian Straßer is an example of the more theoretical papers in argumentation theory that have been presented at CLIMA. The paper introduces 'Sequent-based logical argumentation': a proof-theoretic view on argumentation, where Gentzenstyle sequents are used to represent arguments and attacks are mirrored by particular sequential elimination rules. A comparison with the sequent-based approach to logical argumentation due to Pollock is provided. The proposed method also exemplifies how techniques and methodologies from proof theory can be applied in the context of argumentation theory.

Finally, the work by Adam Wyner, Trevor Bench-Capon, Paul Dunne and Federico Cerutti addresses a central aspect of abstract argumentation, namely instantiation. Their article, entitled 'Senses of "argument" in instantiated argumentation frameworks' presents a new method to instantiate from ASPIC + theories where abstract arguments are used to represent literals and rules (instead of complex arguments, subarguments, etc.). The authors show that this method leads to a more efficient evaluation, but also allows for a better reflection of different terminological meanings of arguments in the instantiated framework.

\section{Acknowledgements}

We thank all reviewers of CLIMA XIV, and the reviewers of this special issue: Philippe Besnard, Anna De Liddo, Louise Dennis, Wolfgang Dvořák, Lluís Godo, Thomas Gordon, Sanjay Modgil, Francesco Santini, Giovanni Sartor, and Bas Testerink.

Moreover, we are very grateful to the TARC Editors-in-Chief, in particular Floriana Grasso and Chris Reed, for pursuing this collection and for the pleasant collaboration. We express our deepest appreciation to Pedro Cabalar, the Co-Chair of LPNMR'13, and the local organisers in Corunna, for all their help and support.

Finally, a special thanks to the authors of this volume and to their excellent contributions, without which none of this would have been possible.

\section{Note}

1. http://centria.di.fct.unl.pt/ clima/.

\section{ORCID}

Paolo Torroni (D) http://orcid.org/0000-0002-9253-8638 